\title{
Gamma-linolenic and pinolenic acids exert anti-inflammatory effects in cultured human endothelial cells through their elongation products
}

\author{
Ella Baker, Elizabeth Miles and Philip Calder \\ University of Southampton, Southampton, United Kingdom
}

\section{Abstract}

It is recommended that humans consume fatty fish twice a week to increase dietary intake of eicosapentaenoic acid (EPA) and docosapentaenoic acid (DHA) to achieve long-term health benefits. However current stocks of fish are likely insufficient to meet the needs of humans. Plant-derived polyunsaturated fatty acids (PUFAs) gamma-linolenic acid (GLA) and pinolenic acid (PIN) may provide sustainable land-based sources of bioactive fatty acids.

Anti-inflammatory effects of GLA and PIN were compared to EPA and DHA in cultured EA.hy926 cells. Cells were treated with PUFAs $(10,25$ and $50 \mu \mathrm{M})$ for 48 hours prior to stimulation with tumour necrosis factor for 24 hours. Incorporation of PUFA was measured by gas chromatography; inflammatory responses were measured by ELISA and flow cytometry.

All fatty acids were incorporated into EA.hy 926 cells, after 48 hours, in a dose dependent manner $(10$ and $50 \mu \mathrm{M})$. Pre-treatment with GLA and PIN $(50 \mu \mathrm{M})$ resulted in significant increases in their elongation products, dihomo- $\gamma$-linolenic acid (DGLA) (p< $0.0001)$ from GLA and eicosatrienoic $(E T r A)(p<0.0001)$ from PIN.

Pre-treatment with GLA, PIN, EPA or DHA $(50 \mu \mathrm{M})$ had differential effects depending on fatty acid and cytokine examined. Pre-treatment of EA.hy926 cells with both GLA and PIN resulted in a lower concentration of soluble ICAM-1 ( $p<0.01)$; however EPA and DHA showed greater reduction $(\mathrm{p}<0.0001)$. MCP-1 production was significantly lower after treatment with PIN $(\mathrm{p}<0.05)$, again to a lesser extent than EPA and DHA $(\mathrm{p}<0.0001)$. Pre-treatment with EPA and DHA $(50 \mu \mathrm{M})$ resulted in lower cell surface expression of ICAM-1 ( $p<0.001, \mathrm{p}<0.0001)$, an effect not observed with GLA or PIN.

Anti-inflammatory effects of GLA and PIN were possibly due to their elongation products, and therefore silencing of elongase 5 (ELOVL5) was explored. ELOVL5 siRNA significantly inhibited the production of DGLA and ETrA in EA.hy926 cells pre-treated with GLA and PIN $(50 \mu \mathrm{M})$. Furthermore significant decreases in sICAM-1 and MCP-1 were not seen after pre-treatment with GLA or PIN in ELOVL5 siRNA silenced EA.hy926 cells.

Plant PUFAs (GLA and PIN) demonstrate anti-inflammatory effects in this model using endothelial cells, but are less potent than EPA or DHA. Anti-inflammatory effects of GLA and PIN may be due to their elongation metabolites; DGLA and ETrA.

\section{Conflict of Interest}

There is no conflict of interest 